$$
\operatorname{con} t-870610--14
$$

CONF-870610--14

AN OVERVIEW OF THE ADVANCED PHOTON SOURCE ${ }^{\star}$ DE88 003076

G. K. Shenoy
Materials Science Division, Argonne National
Argonne, IL 60439
and
D. E. Moncton
Advanced Photon Source Project
Argonne National Laboratory
Argonne, IL 50439
and
Exxon Research \& Eng Ineering Co.
Annandale, NJ 08801

August 1987

bsm

\begin{tabular}{|c|}
\hline 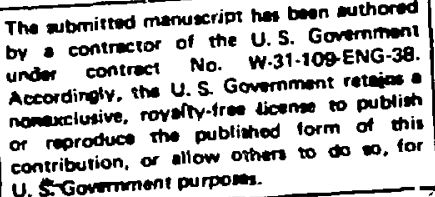 \\
\hline
\end{tabular}

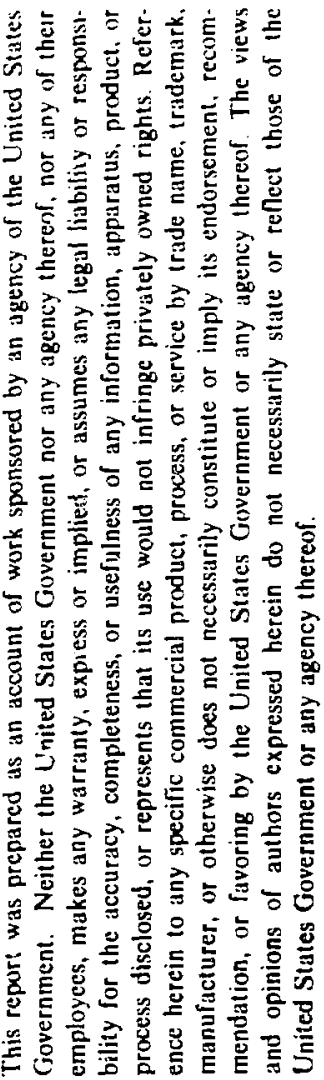

An INVITED TALK presented at the 5th National Conference on Synchrotron

Radiation Instrumentatior, Univ. of Wisconsin-Madison, WI., June 22-25, 1987; to be published as part of the Conference proceedings in Nucl. Inst. and Methods.

\footnotetext{
*This work supported by the U.S. Department of Energy, BES-Materials Sciences, under Contract W-31-109-ENG-38. 


\title{
AN OVERVIEW OF THE ADVANCED PHOTON SOURCE*
}

\author{
G. K. Shenoy \\ Materials Science Division, Argonne National Laboratory \\ Argonne, IL 60439 \\ and \\ D. E. Moncton \\ Advanced Photon Source Project \\ Argonne National Laboratory \\ Argonne, IL 60439 \\ and \\ Exxon Research \& Englneering Co. \\ Annandale, HJ 08801
}

\section{ABSTRACT}

The need for dedicated synchrotron radiation facllities based on insertion devices and a low emittance storage-ring has been recognized for many years. A facility optimized to produce $x$-rays from 1 to $100 \mathrm{keV}$ is expected to dramatically enhance research capabilities in the areas of condense matter physlcs, material sciences, chemical sciences, and biological sciences, in addition to contributing in a major way to the industrial research. This goal w111 be accomplished by the construction of the Advanced Photon Source (APS) facility consisting of a 7-GeV positron storage-ring with $100 \mathrm{~mA}$ current and 35 straight-sections to accommodate insertion devices. The ring energy has been chosen so that a single undulator will provide radiation from 4 to $40 \mathrm{keV}$ (using the first and the third harmonics) with 0.27 bandwidth. The low emittance of $7 \times 10^{-9}$ rad.m will provide hard-x-ray undulator radiation with very high brilliance $\left(\sim 10^{18}\right.$ to $10^{19}$ photons/(s $\left.\mathrm{mm}^{2} \mathrm{mrad}^{2}\right)$. [Construction is expected to begin in 1989 at Argonne National Laboratory.l This overview will mainly address the facility from the users point-of-view.

\footnotetext{
*This work supported by the U.S. Department of Energy, BES-Materials Sciences, under Contract W-31-109-ENG-38.
} 


\section{INTRODUCTION}

During the past decade, electron storage rings have been built specifically dedicated to producing low emittance synchrotron radiation and designed primarily to extract beam lines from the storage-ring bending-magnets. The principal index of performance for these sources is the integrated photon flux.

In the next generation photon sources, the spectral properties of the photon beams are enhanced, firstly through the use of special magnetic devices such as "undulator" and "wigglers", and secondly, through the further reduction of the emfttance of the particle (electron or positron) beam circulating through the storage ring. The design of the 7-GeV Advanced Photon Source (APS) includes these features.

The special magnetic devices, also known as the insertion devices, are placed in the straight sections (or the insertions) of a storage ring. Unlike bending magnets, these sources provide complete flexibility to tailor the characteristics of the radiation to meet the needs of the experiment. The APS, for example, will provide tunable pseudo-monochromatic radiation from the undulators with energles from $1 \mathrm{keV}$ to $40 \mathrm{keV}$, and white radiation from the wigglers to over $100 \mathrm{keV}$.

During the past four years, many natlonal advisory panels have made their highest-priority recommendation the construction of a hard $x$-ray source with features provided by the APS $[1-5]$. The unprecedented brilliance of the hard $x$-rays from the APS is expected to revolutionize many areas of basic research, industrlal research, biological and medical research, and defense-related research; the unique properties of the radiation from the APS undulator will provide new possibilities of imaging at a sub-micron level, perhaps leading the way to holographic lmaging of the molecules and atoms in the future 
sources. The APS will be operated as a national user facłlity serving the entire U.S. $x$-ray research community of several thousand users, with about 300 scientists taking data at any given time.

The present paper glves a brlef overview of the 7-GeV APS from a user point-of-view. A discussion of the storage ring and other accelerator component design have been presented earlier [6].

II. INSERTION DEVICES ON THE APS

The distinction between the two types of IDs - wigglers and undulators is based on the value of the so-called deflection parameter $\mathrm{K}$ given by

$$
K=0.934 \mathrm{~B}_{0} \lambda_{0}
$$

where $B_{0}$ is the peak magnetic field (Tesla) In the device and $\lambda_{0}$ is the spatial pertod $(\mathrm{cm})$ of the magnetic structure.

W!en $\mathrm{K}$ is small $(<4)$, the device is called an undulator. The radiation from various poles of an undulator interferes construciively resulting in a spectrum consisting of sharp peaks at harmonics of the first-harmonic energy, $E_{1}$. This energy is given by

$$
E_{1}(k e V)=\frac{0.95 \mathrm{E}^{2}\left(\mathrm{GeV}^{2}\right)}{\lambda_{0}(\mathrm{~cm})\left(1+\mathrm{k}^{2} / 2\right)}
$$

where $E$ is the positron energy. The following ise important features of APS undulators: 
The undulators on the APS ring to be built with the available Nd-Fe-B hybrid magnet technology will deliver first-harmonic radiation with energles ranging from a few hundred eV to $20 \mathrm{keV}$. The typical on-axis angle Integrated brilliance for the fundamental radiation ranges from $10^{18}$ to $10^{19}$ photons $/ \mathrm{s} / 0.1 \% \mathrm{BW} / \mathrm{mrad}^{2} / \mathrm{mm}^{2}$.

- A fully tunable undulator with $3.3 \mathrm{~cm}$ period on the APS will deliver radiation from 4 to $14 \mathrm{keV}$ in the first-harmonic and from 12 to $42 \mathrm{keV}$ in the third-harmonic. The energy tunability is achieved by varying the undulator gap from $1.0 \mathrm{~cm}$ to about $2.5 \mathrm{~cm} \mathrm{[7].}$

For applications needing only the high-energy undulator fundamental radiation, device with a perlod of $2.3 \mathrm{~cm}$ will be tunable from 13.5 to $20 \mathrm{keV}$ through gap variation.

- The phase space dimensions of the photon source is nearly equal to that of the positron beam for the hard x-rays delivered by the APS undulators.

The vacuum chambers for the undulators will permit a minimum gap of either $1.4 \mathrm{~cm}$ or $1.0 \mathrm{~cm}$. The larger chamber will be used during the Intial operational period of the APS.

When $K$ is large $(>10)$, the device is called a wiggler. The radiation peaks from higher harmonics of a wiggler tend to smooth out due to smearing effects of the positron-beam emittance and energy spread. This results in a smooth spectrum similar to that from a bending magnet (BM). The intensity of 
the radiation from a wiggler with $\mathrm{N}$ periods is approximately equal to an Incoherent sum of intensities from $2 \mathrm{~N}$ poles. One can also increase or decrease the critical energy of the photons from a wiggler by proper cholce of $\mathrm{B}_{\mathrm{o}}$, while maintaining a large value of $\mathrm{K}$ given by $\mathrm{Eq} \cdot(1)$. The photon critical energy for the APS operated at $7 \mathrm{GeV}$ is given by

$$
E_{c}(k e V)=32.59 B_{0} \text {, }
$$

and the horizontal opening angle of the radiation is given by

$$
\theta_{\mathrm{H}}= \pm 73 * \mathrm{~K} \text { Hrad. }
$$

Several important features of the APS wigglers deserve mention:

They will rarely require magnetic flelds produced by superconducting magnets. The fleld provided either by the Nd-Fe-B hydrid magnets or by electromagnets is adequate to obtain large $K$ values and nearly smooth photon energy spectrum, up to the highest photon energies generally useful for $x$-ray research.

- The value of $\theta_{H}(\mathrm{Eq} .(4))$ for the APS wigglers is approximately \pm 1 mrad. Hence, the APS wigglers deliver very high brightness hard $x$-rays compared with those on the low-energy storage rings. 
The avallability of $6 \mathrm{~m}$ long straight sections, is suitable for longperiod wigglers designed to provide radiation of low critical energy. These devices (with $K \sim 10$ ) operate with $B \approx 10 \mathrm{~m}$ and hence provide a low divergence photon beam. These high-brightness wigglers are unique to the present design and are essentlal for many research programs.

In many investigations, there is a need for radiation with linear polarization alon; the vertical axis. A design of such a highbrightness wiggler with hybrid magnet configuration has been completed.

The IDs with $4<K<10$ produce energy spectra with features that are Intermediate between those of undulators and wigglers. Devices capable of delivering hard $x$-rays from the undulator to the wiggler reglme through a gap variation have been designed for the APS.

In Table 1, a set of optimized parameters for a typlcal wiggler and an undulator for the 7-GeV APS are presented. Their energy spectra are shown in Fig. 1. The undulator curve represents the envelope of the peak of the firstand third-harmonic energy spectra when the undulator described in Table 1 is tuned by varying its gap from $1 \mathrm{~cm}$ to $2.8 \mathrm{cms}$. A single undulator thus provides high-brilliance-radiation from roughly 4 to $40 \mathrm{keV}$ using the first and the third harmonics. A comparison has been made of the radiation brilliance from other important sources.

\section{PHOTON SOURCE DIMENSIONS}

The spectral brilliance, the quantity which is optinized in the APS design, is the spectral intensity emitted in the unit phase-space volume of the radiation field. Hence, it is essential to minimize the phase-space 
volume. For the APS in the hard $x$-ray range, the phase-space volume of the photon field is totally determined by the positron phase-space volume. In terms of the betratron functions, $\beta_{x}$ and $\beta_{y}$, in the horizontal and the vertical directions, the rms Gaussian widths and divergences of the positron beam are given by

$$
\sigma_{1}=\sqrt{\varepsilon_{1} \beta_{i}} ; \quad \sigma_{i}^{\prime}=\sqrt{\varepsilon_{1} / \beta_{1}} ; 1=x, y
$$

where $\varepsilon_{\mathrm{x}}=7.3 \times 10^{-9} \mathrm{rad} \cdot \mathrm{m}$ and $\varepsilon_{\mathrm{y}}=7.3 \times 10^{-10} \mathrm{rad} \cdot \mathrm{m}$ are the horizontal and vertical emittances. The phase-space volume is given by $S=4 \pi \sigma_{x} \sigma_{y} \sigma_{x}{ }^{\prime} \sigma_{y} ' \cdot$

The source size, however, can be altered by changing the value of betatron functions. Their values can be optimized for the best performance of the lattice, but it is advantageous to have certain flexibility in choosing their values in the dispersion-free straight sections. Thus having small values of betatron functions will produce small physical size of the source but large divergence. Small betratron values are ideal for wigglers. In the undulator straight sections, small values of the betatron functions will produce broad energy peaks and higher intensity in the region of the higher harmonics. Such a spectral distribution is useful for certain applications, such as anomalous scattering. On the other hand, many investigations demand small angular divergence of the undulator beam and sharper peaks in the energy spectrum. For these cases, it is appropriate to use larger values of betatron function. It should be pointed out that because of smaller emittance of the lattice in the vertical direction, the vertical angular divergence is usually small. Hence, it is adequate to increase the value of $B_{x}$ to achieve small aivergence photon beams. 
In Table 2, some typical values of the betatron functions that are possible in the various parts of the APS lattice are given along with the resulting source dimensions.

\section{LAYYUT OF STORAGE-RING AND EXPERIMENTAL FACILITIES}

In Fig. 2, the present layout of the storage-ring and other accelerator components is shown. A $200 \mathrm{keV}$ linear accelerator produces 3 amp electron pulses of 16.5 nsec width. This beam is converted to a beam of $25 \mathrm{~mA}$ positron pulses using a tungsten target and then accelerated to $450 \mathrm{MeV}$. The positrons are further accelerated to $7-\mathrm{GeV}$ in a booster synchrotron $367 \mathrm{~m}$ clrcumference. The accelerated positrons are then injected into pre-defined number of the 1248 available rf bunches in the storage ring. Typical number of bunches is expected to be about 40-60 to store $100 \mathrm{~mA}$ current. The parameters of the storage ring are given in Table 3. One of the 40 sectors of the storage-ring is shown in Fig. 3 to indicate the location of the insertion device and bending magnet sources. Note that although there are 80 bending magnets in this lattice, only 35 will be available for extracting photon beams.

The photon beams from the IDs located in the 34 avaflable stralght sections emerge through the beam ports in the vacuum chamber at the downstream ends of the dipoles. The beams travel in vacuum pipes out of the storage ring shielding tunnel into the experimental hall. Each beam is generally mantpulated a number of times with various types of optical elements before reaching the sample for the experiment. The beam lines and experiments, some of which may extend more than $80 \mathrm{~m}$ in length, occupy the annular floor space around the storage Iing. To accommodate $80 \mathrm{~m}$ beams, the radial wicth of the experiment hal1 is about $27 \mathrm{~m}$. It is expected that some extra-long beam lines will 
extend beyond the outside wall of the experiment hall and will be housed in special extended eiclosures. A partial layout of the experiment building showing the photon beams is given in Fig. 4.

Generally speaking, the photon beam line consists of four functional sections. The first section is the ID on the storage ring straight section that provides the radiation source. In some cases, the bending magats deliver the radiation.

The second section, immediately outside the storage ring but still inside the concrete shielding tunnel, is the front-end section. This section contains safety shutters, masks, and other components to define the energing x-ray beam.

The third section contains hard $x$-ra optics. In a majority of experiments, this assembly delivers a focused monochromatic beam of $x$-rays. The section includes crystals and/or mirror optics that are designed to handle the radiation power loads and provide the monochromatization required for the specific investigation.

The monochromatic $x$-rays are delivered to an experiwental station that forms the fourth section. This station cortains the sample under investigation and detectors and/or analyzers to detect and characterize the scattering, Imagling, and absorption processes.

Most of the beam lines, including the experimental setups, will be shorter than 80 , and can be housed in the experimental hall. The entire 27-m-wide annular experiment hall is serviced by 5-ton overhead cranes and by several clusters of offices, laboratories, clean rooms, experiment staging rooms, etc., distributed along the periphery.

Number of speclalized beam line factlities will be built at the APS. One example is a special beam line facility for coronary angiograrity studies. In 
view of the encouraging results of recent anglography tests at Staniord using synchrotron Iadiation, further research using a dedicated bean at the APS wili be of unique value for developing these techniques and for extending the use of the synchrotron radiation into new realms of medical examination and diagnosis.

Another example concerns speciallzed facilities for classified research. In view of the $\mathrm{DOE}$ intent that classified research be carried out at the APS if the need arises, we have considered various means for carrying out such work effectively, with minimal impact on other activities at the APS.

The central laboratory/ofice bullding is a three-story building with $\sim 180,000 \mathrm{ft}^{2}$ total floor area (exclusive of auditorium), located adjacent to the experiment hall as shown in Fig. 2. The central laboratory/office buildIng houses the regular operations staff, control room, clean room, staging areas for major component assemblies, computer facilities, auditorium, library, machine shops, and various other service in addition to users. In addition, there are laboratory/ofifice modules which will be avallable for the users located around the outer perimeter of the experimental hall (see Fig. 2). The detailed layout of this user area is now being finalized.

Convenient housing for the many outside users of the source will be an Important component of the overall facility. Curzent predictions of user numbers indicate an initial need for a 240-bed complex. It would be highly desirable to have this housing completed approximately one year before the source is user-read: to be avallable to research groups constructing beam lines prior to commissioning. 


\section{ACKNOWLEDGMENTS}

We would like to thank the entire staff of the Advanced Photon Source which has contributed to the design discussed in this paper. 
TABLE 1

Optimized Parameters of a Nd-Fe-B Hybrid Insertion Devices with the APS

\begin{tabular}{lll}
\hline Parameters & Undulator & Wiggler \\
\hline Periods, $\lambda_{0}(\mathrm{~cm})$ & 3.3 & 15.0 \\
Magnet Gap Range, G (cm) & $1.0-2.8$ & 3.7 \\
Peak Field Range on Axis, B (T) & 0.78 to 0.21 & 1.0 \\
K value range & 2.4 to 0.65 & 14 \\
Lesgth of Straight Section (m) & 6.0 & 0.4 \\
Minimum Length of Transition Section (m) & 0.4 & 5.2 \\
Maximum Length Avallable for the & & 10 \\
Insertion Derice (m) & 5.2 & 32.6 \\
Maximum Undulator Periods, N & 162 & $*$ \\
Critical Energy (keV) & & \\
\hline
\end{tabular}

${ }^{*}$ Tunability range given in the text. 
13

TABLE 2

Typical values of betatron functions in the different parts of the lattice and the dimensions of different sources

Source

\begin{tabular}{llllll}
$\beta_{x}$ & $\beta_{y}$ & $\sigma_{x}$ & $\sigma_{y}$ & $\sigma_{x}^{\prime}$ & $\sigma_{y}^{\prime}$ \\
$m$ & $m$ & $\mu m$ & $\mu m$ & $\mu r a d$ & $\mu \mathrm{rad}$ \\
\hline 5.5 & 13.0 & 200 & 97 & 36 & 8 \\
13.0 & 10.0 & 308 & 85 & 24 & 9 \\
20.0 & 5.0 & 382 & 60 & 19 & 12 \\
13.0 & 10.0 & 308 & 85 & 24 & 9
\end{tabular}




\section{REFERENCES}

1. P. Eisenberger and M. L. Knotek, Chairmen, "Planning Study for Advanced National Synchrotron-Radiation Facilities," Sandia National Laboratory Report (March 1984).

2. F. Seitz and D. Eastman, Chairmen, "Major Facilities for Materials Research and Related Disciplines," National Acadeny of Science, Washington, D.C. (1984).

3. W. Brinkman, Chatrmen, "Physics Throughout the 1990's: An Overview," NRC Report, National Academy of Sciences, Washington, D.C. (1986).

4. F. Stehl1, Chairman, "Review of the National Research Council Report on Mafor Facilities for Materials Research," Energy Research Advisory Board Subcommittee, DOE (1986).

5. B. Ancker-Johnson, Chatrperson, "Review of the National Research Council Report on Physics through the 1990's," Energy Research Advisory Board Subcommittee, DOE (1987).

6. Y. Cho, Particle Accelerator Conference, Washington, D.C., March 16-19, 1987 .

7. P. J. Viccaro and G. K. Shenoy, presented at this conference. 


\section{FIGURE CAPTIONS}

Fig. 1 Brilifance of various sources as a function of energy. The 7-GeV APS undulator is the $3.3 \mathrm{~cm}$ period device discussed in the text. The curve represents the envelope of the first and the third harmonic energies tunable by the gap variation. Similar curves for the undulators on the 1-2 GeV Synchrotron Radiation Source at Berkeley, X-1 undulator on the National Synchrotron Light Source (NSLS) and for the $7.7 \mathrm{~cm}$ period undulator on PEP ring (14.5 GeV) at Stanford are also shown.

Fig. 2 Layout of the Advanced Photon Source showing various conventional construction.

Fig. 3 One cell of the storage ring magnet lattice showing the location of the insertion device and bending magnet sources.

Fig. 4 Layout of typical beamlines in a part of the storage ring experimental hall. These beamlines are defined for a possible set of investigations and use undulator, wiggler and bending magnet sources. 


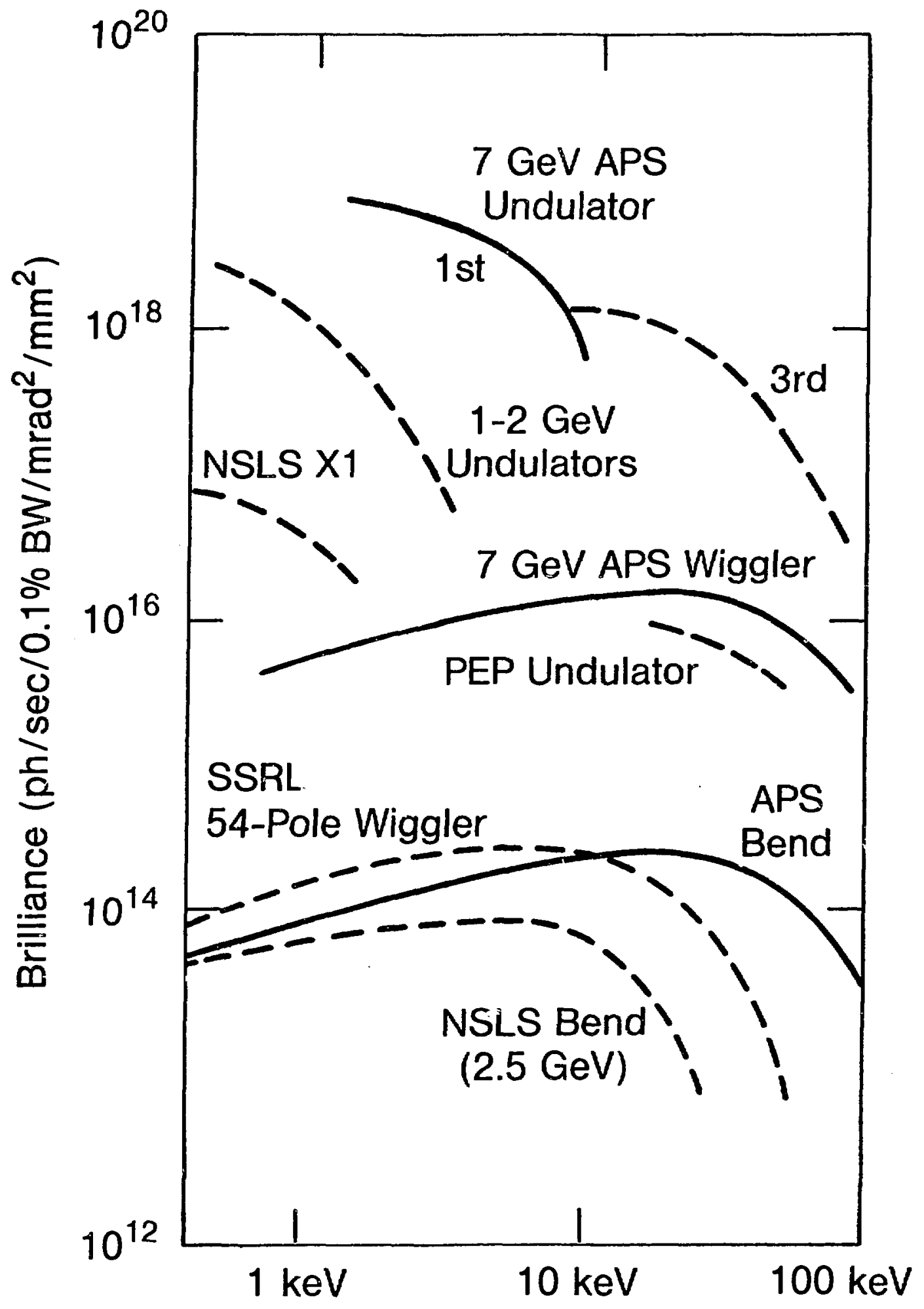




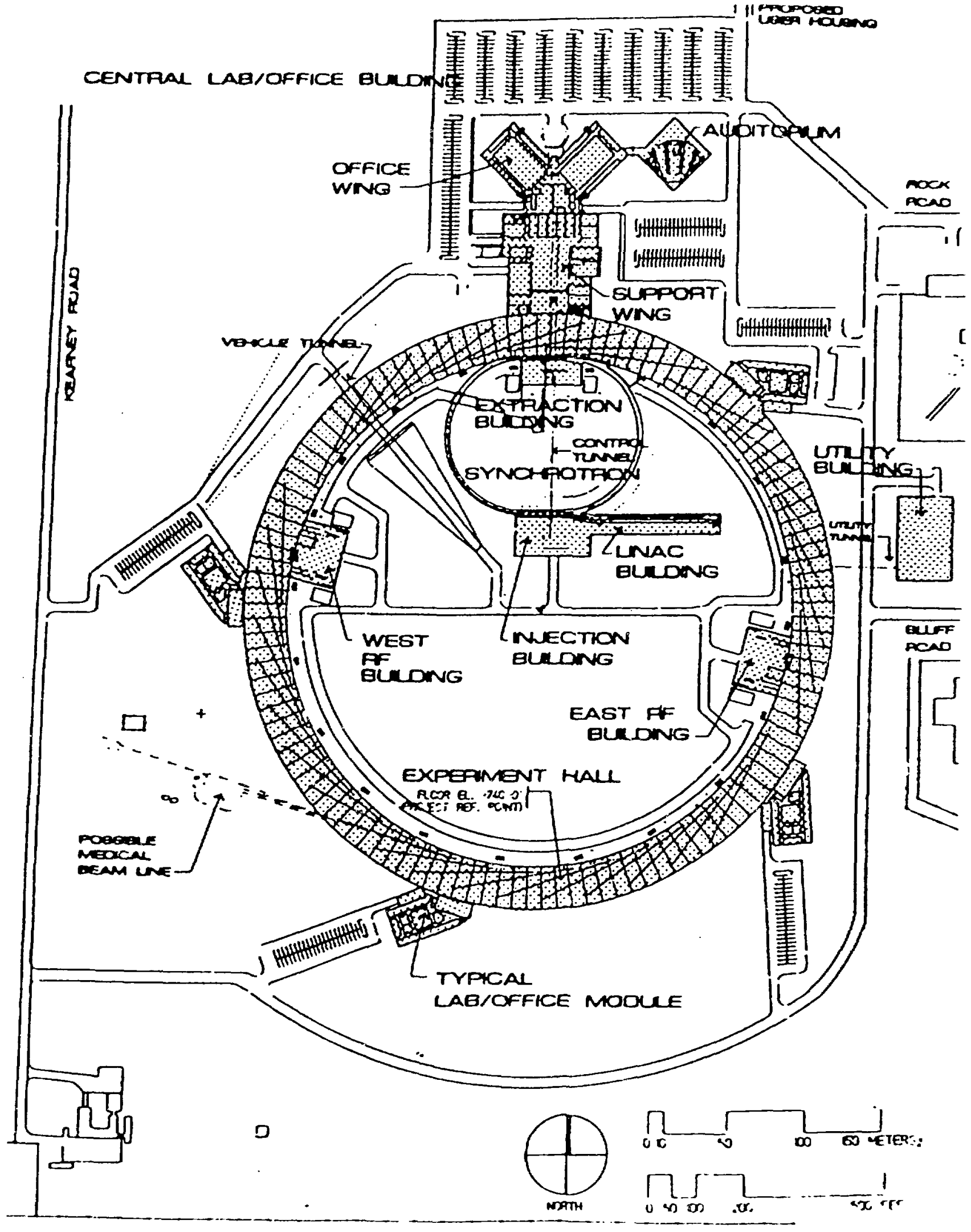




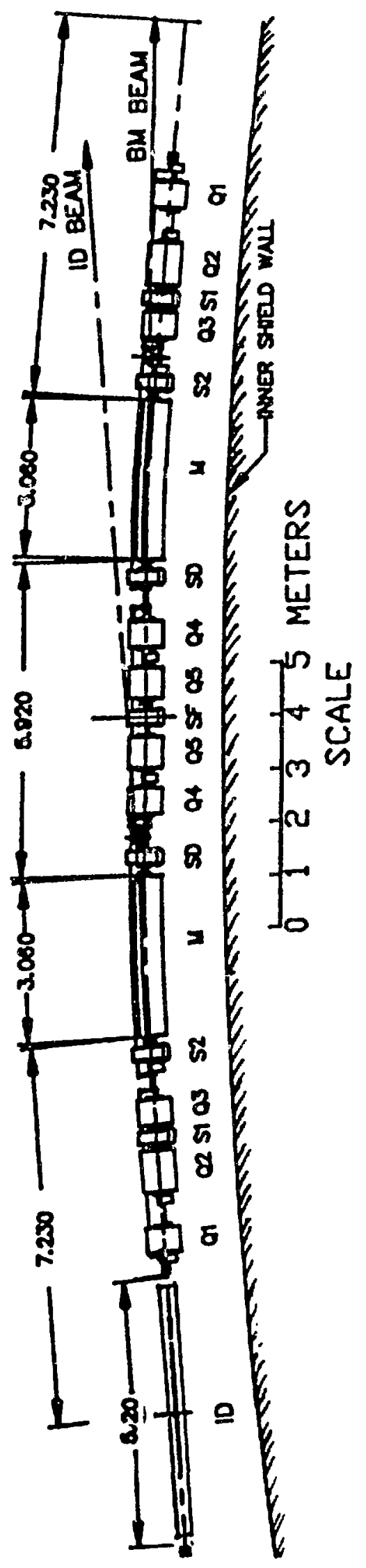




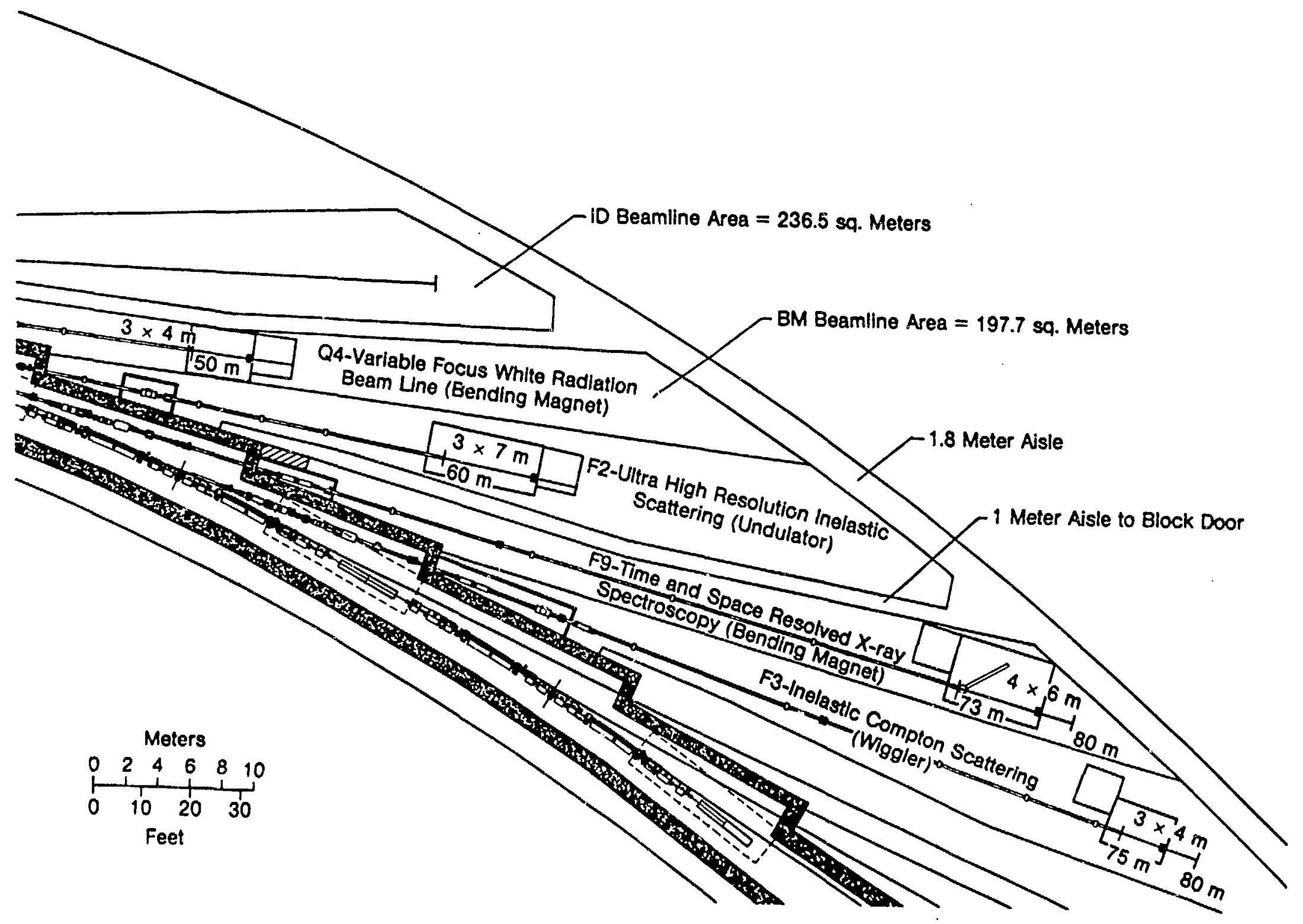

